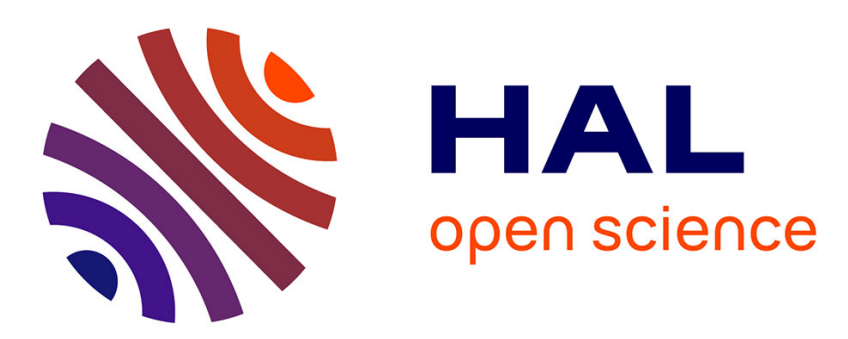

\title{
Using VHF navigation aid to estimate radar cross section of airliners by inverting the radar equation
}

Stéphane Saillant, Philippe Dorey, Sylvain Azarian

\section{To cite this version:}

Stéphane Saillant, Philippe Dorey, Sylvain Azarian. Using VHF navigation aid to estimate radar cross section of airliners by inverting the radar equation. RADAR2018, Aug 2018, BRISBANE, Australia. hal-02183150

\section{HAL Id: hal-02183150 \\ https://hal.science/hal-02183150}

Submitted on 15 Jul 2019

HAL is a multi-disciplinary open access archive for the deposit and dissemination of scientific research documents, whether they are published or not. The documents may come from teaching and research institutions in France or abroad, or from public or private research centers.
L'archive ouverte pluridisciplinaire HAL, est destinée au dépôt et à la diffusion de documents scientifiques de niveau recherche, publiés ou non, émanant des établissements d'enseignement et de recherche français ou étrangers, des laboratoires publics ou privés. 


\title{
Using VHF navigation aid to estimate radar cross section of airliners by inverting the radar equation
}

\author{
Stéphane Saillant, Philippe Dorey, Sylvain Azarian \\ ONERA \\ The French Aerospace Lab \\ Palaiseau, France \\ stephane.saillant@onera.fr, philippe.dorey@onera.fr, sylvain.azarian@onera.fr
}

\begin{abstract}
The VHF Omnidirectional Range (VOR) navigation aid system can be used as donor of signal for passive radar experiments. An experiment was set up close to a VOR beacon to detect airliners and to analyze their radar cross section in function of their attitude. This paper presents the configuration of the trials, the adapted signal processing and the detection results obtained for one of the detected targets. The radar cross section (RCS) was estimated from the computation of power budget in radar equation. An analysis of the evolution of the RCS is given in function of the attitude of this detected target in azimuth and elevation. Mean values of RCS for many models of airliners detected during the trial campaign and deduced by the same approach are then presented.
\end{abstract}

Keywords - passive radar, bistatic, VOR, VHF, radar cross section

\section{INTRODUCTION}

In the frame of studies about passive radar conducted at ONERA, we proposed to use some sources of opportunity like VOR (VHF Omnidirectional Range) beacons for the detection of air targets.

VOR transmitters have already been used as donor of signal for different kinds of passive radar for the detection of airliners [1].

One new experiment was set up in South of France close to a VOR beacon in order to detect non-cooperative air targets and to estimate their bistatic cross section.

This paper will present the configuration of trials, the adapted signal processing and the results of detection obtained for airliners which are present in the close aerial space. Their evolution of radar cross section RCS is given in function of their attitude in azimuth and elevation.

\section{CONFIGURATION OF TRIALS}

\section{A. Geographical configuration}

The experiment took place in South of France near Marseille as shown on figure 1. The VOR transmitter used for the trials is the beacon located close to Martigues. This station is 650 meters of altitude. The receiving system was set up at $17 \mathrm{~km}$ from there in Port-Saint-Louis-de-Rhone at the level of the sea.
The configuration of the system is like a bistatic passive radar. The two sites for transmitting and receiving are separated of $20 \mathrm{~km}$.

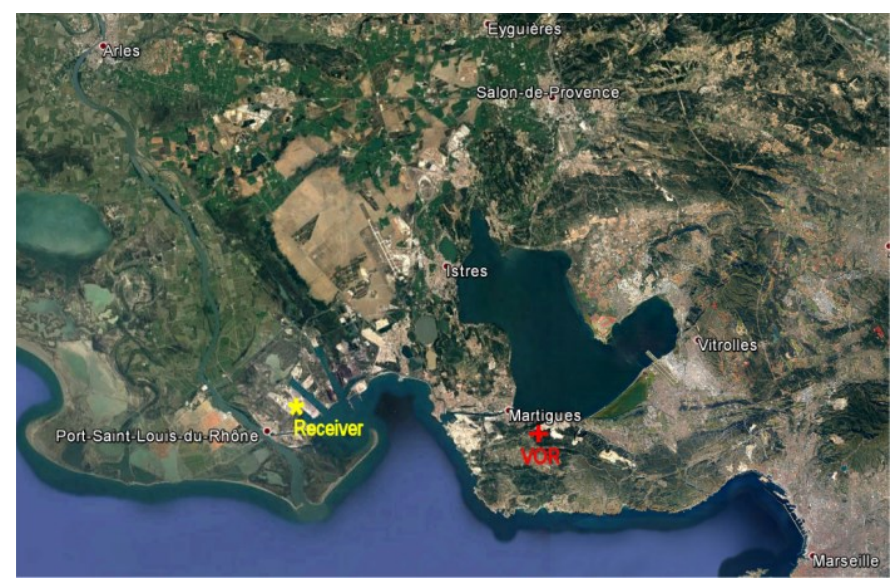

Figure 1 - Bistatic radar configuration (Tx: VOR transmitter site in red, Rx: receiving site in yellow)

\section{B. The VOR transmitter}

One VOR station is a radio positioning system used for aerial navigation. It is a transmitter of VHF frequencies. This beacon can transmit a power of 200 Watts in the frequency bandwidth between $108 \mathrm{MHz}$ to $117.95 \mathrm{MHz}$.

The VOR beacon in Martigues operates at the carrier frequency $(\mathrm{Fe})$ of $117.3 \mathrm{MHz}$, horizontally polarized in order to reduce the influence of the ground on the radiation pattern. This signal is modulated $30 \%$ in amplitude by a $9.960 \mathrm{kHz}$ subcarrier itself frequency modulated by a signal of $30 \mathrm{~Hz}$. The spectrum of the signal consists mainly in fixed frequency lines (as presented on Figure 2).

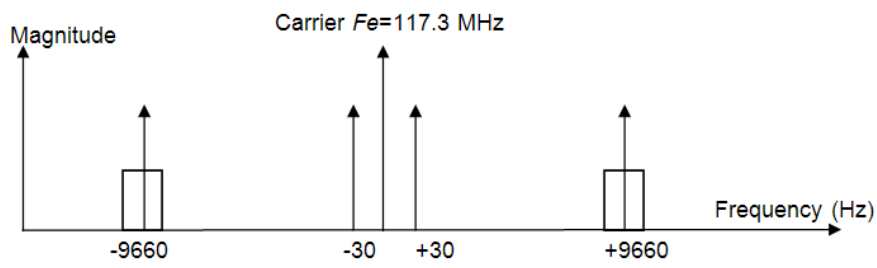

Figure 2 - Schematic diagram of the spectrum of VOR signal transmitted by Martigues station 


\section{Receiving system}

The receiving system consists of one horizontal antenna, a tuned receiver and a data acquisition system. The received signal is digitalized at the rate $F_{\text {num }}=192 \mathrm{kHz}$ around $F e$, the transmitting frequency carrier of the beacon.

A receiving item of information about the flights of airliners through the automatic dependent surveillancebroadcast protocole (ADS-B) is used to get the positions of the planes in real-time in the nearby area at the moment of the measurements. This allows us to verify that the detections carried out after processing are related to known trajectories and thus to analyze radar cross section according to the type of target.

\section{Experimental conditions}

The passage of a target is represented as on the Figure 3.

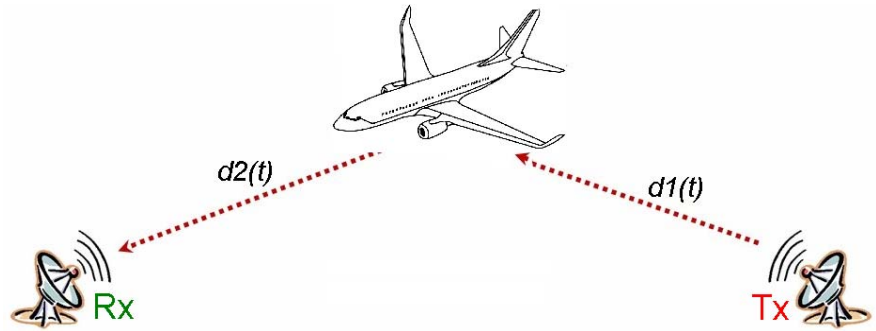

Figure 3 - Principle of bistatic radar

Viewed from the receiver, at each time $t$, a target is seen at the bistatic distance $d_{R}(t)$ with $d 1(t)$, the distance from transmitter to target and $d 2(t)$ from target to receiver.

$$
d_{R}(t)=d 1(t)+d 2(t)
$$

We consider $d_{m R}(t)=d_{R}(t) / 2$, the average distance equivalent to the target distance for a monostatic radar. For a given target, this distance $d_{m R}(t)$ is known from the trajectory information provided by the ADS-B receiver.

At each instant $\mathrm{t}$, the speed $v_{R}(t)$ is calculated as:

$$
v_{R}(t)=\frac{d}{d t}\left(d_{m R}(t)\right)
$$

From the receiver, the target is seen at each instant at the Doppler frequency $f_{d}(t)$ given as

$$
f_{d}(t)=-2 v_{R}(t) / \lambda
$$

with $\lambda=\mathrm{c} / \mathrm{Fe}$, the wavelength of the carrier.

It is recalled that the VOR signal transmitted by the beacon consists mainly of fixed frequency lines. The bandwidth carried by this continuous signal cannot be used to locate one target in distance by correlating the received signal with the transmitted code. Indeed the capacity to localize the plane by this technique is not enough efficient with a range resolution around $15 \mathrm{~km}$.

A FFT (Fast Fourier Transform) is then be performed on the received signal to detect the targets in the frequency domain at their respective Doppler frequencies $f_{d}(t)$.

\section{SIGNAL PROCESSING}

\section{A. Doppler processing}

For targets detection, the received signal is integrated by performing one FFT over a duration of one second i.e. a coherent integration time $T_{i}=1$ second. This operation is thus repeated every second.

A 3-D image is then calculated (Figure 4) where the evolution of the Doppler frequency shift due to the motion of the target is plotted as x-axis, in function of the time (as yaxis) and amplitude (the color scale).

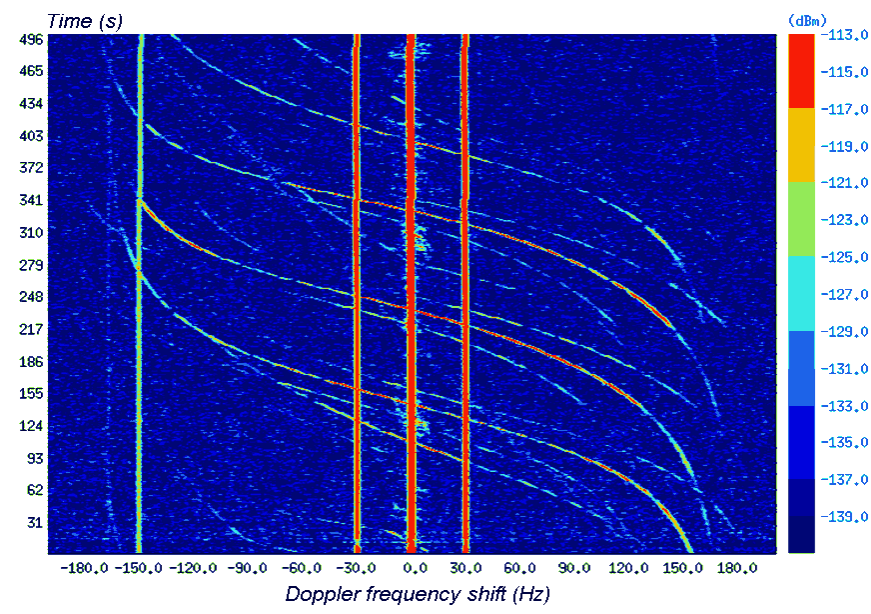

Figure 4 - Evolution of Doppler vs time

The color level indicates the signal amplitude in $\mathrm{dBm}$. The image is normalized with a lower value which is the average noise level. The frequency domain is limited to the maximum value of the Doppler which can be observed for the fastest airliners.

This image (Figure 4) was obtained for an overall processing time of 500 seconds i.e. 500 integration time cells. It shows numerous traces which characterize the variations over time of the Doppler of airliners flying in the nearby area. It can be noticed also the presence of fixed lines. The fixed lines at $0 \mathrm{~Hz}$ correspond to the direct path of the carrier and the 2 lines of modulation appear around at $+/-30 \mathrm{~Hz}$.

The Doppler frequency shift of one target $f_{d}$ is also characterized by 3 lines which evolve with time as one main trace surrounded by 2 symmetrical lines at $f_{d}-30 \mathrm{~Hz}$ and $f_{d}+30$ $\mathrm{Hz}$.

In this example (Figure 4), we also observe the presence of 2 other fixed lines at $-150 \mathrm{~Hz}$ and $-170 \mathrm{~Hz}$, which correspond to jammers present in the frequency band of the receiver.

\section{B. Images cleaning}

In order to work on cleaner images, the fixed lines associated to the direct path with the known Doppler frequencies $(-30,0,+30)$ are simply suppressed with a gabarit of few $\mathrm{Hz}$ around each line.

The cancelation of the two lines at $+/-30 \mathrm{~Hz}$ modulations surrounding the main trace of one target is realized at each integration time cell through the detection of successive maximums. When a main plot of a target is detecting, the 
amplitudes of modulation lines at $+/-30 \mathrm{~Hz}$ from either side are brought back to noise level.

All other interferences are cleaned by applying a method of histogram thresholding.

A clean image is then obtained on which the main traces of detected targets are kept (as shown on the Figure 5).

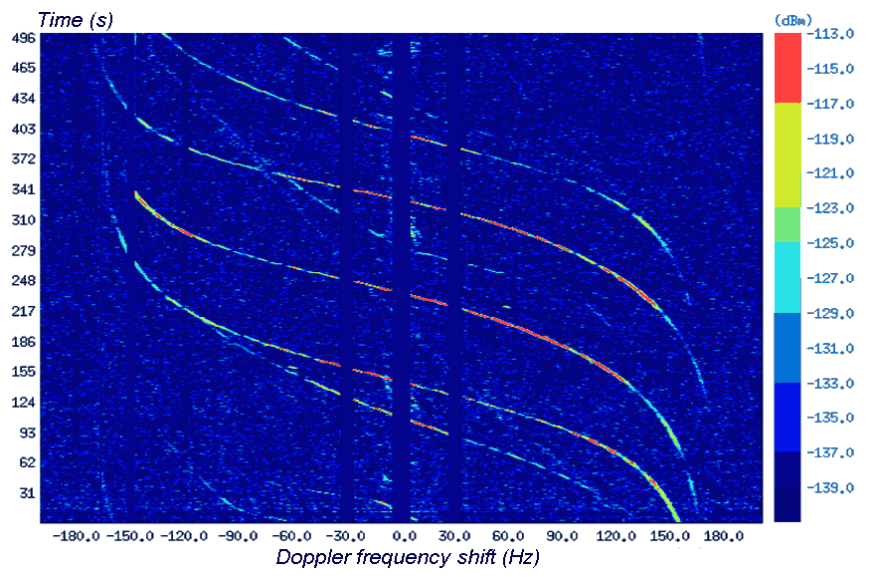

Figure 5 - Clean image of Doppler vs time

It can be observed that fixed lines of jammers, carrier and modulations have been well suppressed. This allows us to extract the good amplitude of potential target echoes. The amplitude of these echoes is obviously not exploited in the erased areas.

\section{Extraction of target echoes}

For this experiment, 70 ADS-B recordings of aircrafts trajectories flying in the area of the VOR station are available. In order to illustrate the method of extracting the target echoes, we present the example of the trajectory of one aircraft which presents a sufficient size to be detected with a high level of signal-to-noise ratio $S N R$.

This target is one airbus A321, for which we have the complete trajectory in the time window of the experiment duration.

\section{1) Trajectory}

The next Figure 6 shows the evolution over time of the latitude and the longitude of the A321 airplane.

The color indicates its progression over time for this passage from North-East to South-West.

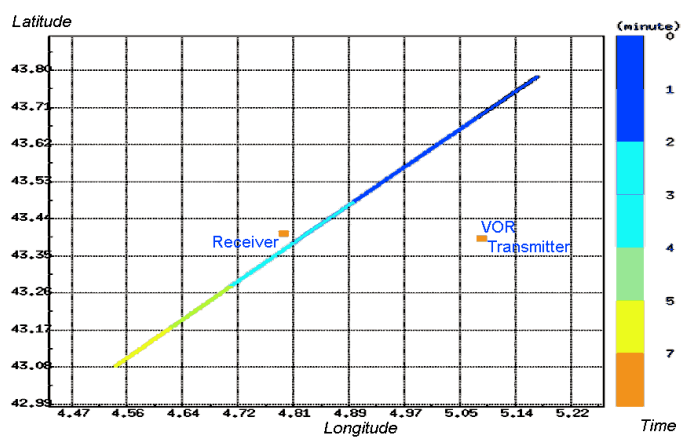

Figure 6 - Trajectory of A321 airplane chosen as target of interest
As the geographic coordinates of the transmitter (VOR) and the receiver are known, the two distances of the target can be calculated from ADS-B positions:

- $\quad d 1(t)$, from VOR transmitter-to aircraft,

- $\quad d 2(t)$, from aircraft to the receiver.

The evolutions of these distances in function of time are presented on the Figure 7.

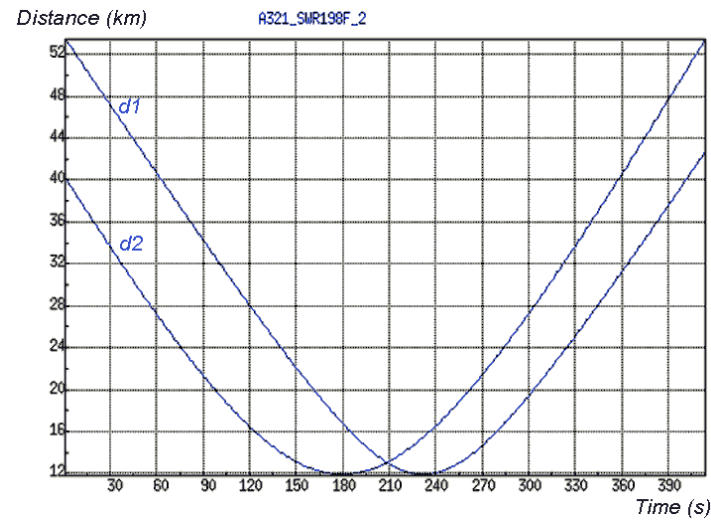

Figure 7 - Evolutions of bistatic distances of the target A321 SWR198F d1: Transmitter to target, $\mathrm{d} 2$ : Target to receiver

The mean bistatic distance $d_{m R}(t)$ is also presented on the following Figure 8.

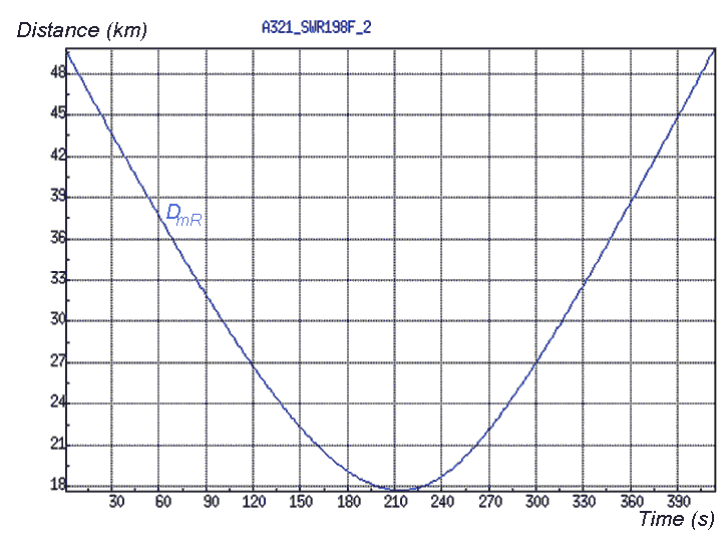

Figure 8 - Mean bistatic distance for the target A321 SWR198F

The Doppler shift due to the motion of the target can thus be calculated as:

$$
f_{d}(t)=\frac{d}{d t}\left(d_{m R}(t)\right)
$$

The theoretical evolution of the Doppler of the considered target A321 SWR198F during its passage in the field of detection is plotted on the Figure 9. 


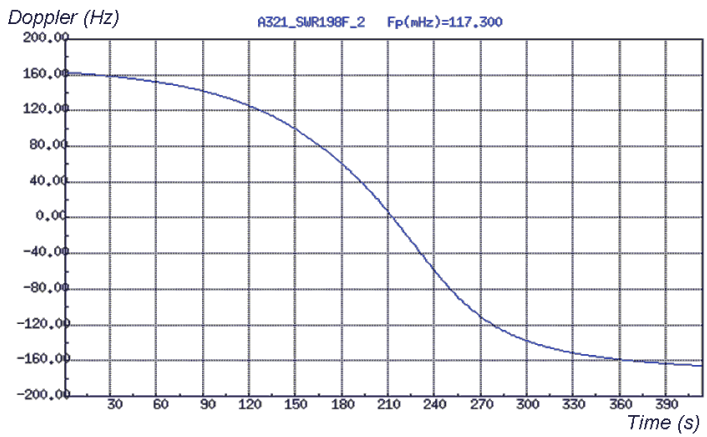

Figure 9 - Evolution of the Doppler of A321 target at $117.3 \mathrm{MHz}$, the operating frequency of the VOR station

With the ADS-B information about the time of passage of the target, a gauge around the expected Doppler trace with 2 curves flanking $f_{d}(t)$ at $+/-15 \mathrm{~Hz}$ can be superimposed on the Doppler-time-amplitude image calculated by Doppler processing as shown on the Figure 10 (white lines reported on the image).

It can be noticed that the airplane is perfectly detected around the theoretical Doppler value $f_{d}(t)$ calculated previously.

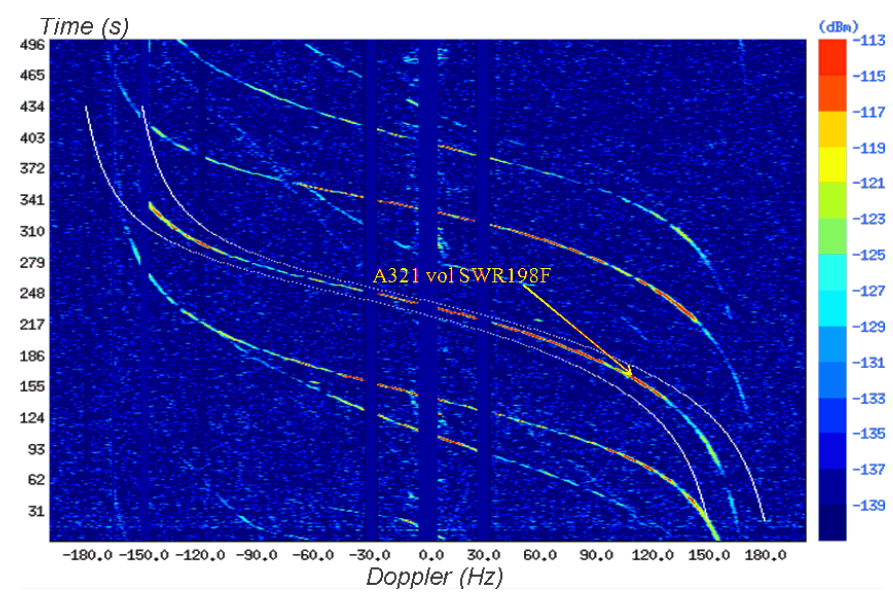

Figure 10 - Different Doppler traces of different targets versus time White lines represent the gauge of expected Doppler for the considered target

This confirms that the extraction of the amplitude values of these echoes over time could be effectively attributed to our target of interest (the airbus A321 SWR198F).

\section{2) Extraction of detected plots}

The amplitude $A(t)$ of the target echo is extracted around the theoretical value of the Doppler $f_{d}(t)$. The average noise level $B(t)$ is also calculated. A threshold of $13 \mathrm{~dB}$ above the average noise is considered. The amplitude of extracted echoes should verify:

$$
A(t) \geq B(t)+13 d B
$$

The following Figure 11 shows the result of this extraction. A line displayed in red at the upper part of the graphic is representing the duration of the flight path of the aircraft. In light blue the value of the amplitudes of the echo detected with the minimum signal-to-noise ratio of $13 \mathrm{~dB}$.

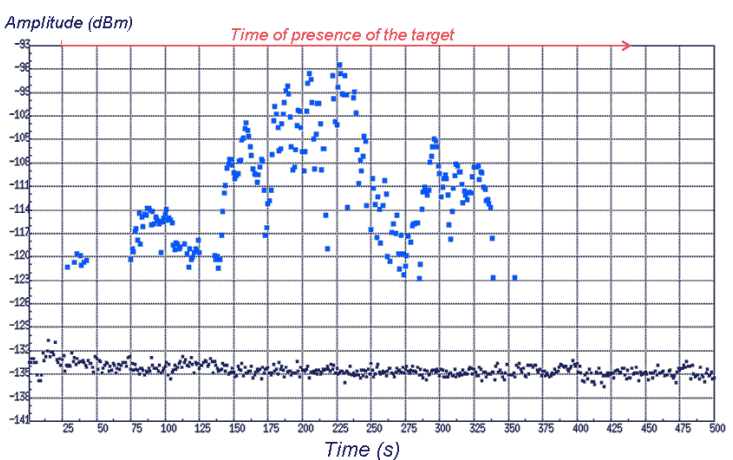

Figure 11 - Detected plots of the target during its passage (light blue dots) and mean noise level (dark blue dots)

\section{3) Radar Cross Section calculation}

In the case of a bistatic radar, the level received from the target is expressed as follow:

$$
\operatorname{Pr}=\frac{P e \cdot G e(\theta e, \psi e) \cdot G r(\theta r, \psi r) \cdot \sigma \cdot \lambda^{2}}{L \cdot(4 \pi)^{3} \cdot D e^{2} \cdot D r^{2}}
$$

with:

- $\operatorname{Pr}=$ power of the received signal,

- $P e=$ power transmitted,

- $G e(\theta e, \psi e)=$ transmitting antenna gain in the direction $(\theta \mathrm{e}, \psi \mathrm{e})$ with:

$\circ \theta \mathrm{e}=$ target azimuth in transmitter reference,

○ $\quad \psi \mathrm{e}=$ target elevation angle in transmitter reference,

- $G r(\theta r, \psi r)=$ receiving antenna gain in the direction $(\theta r, \psi r)$ with:

$\circ \quad \theta \mathrm{r}=$ target azimuth in receiver coordinates,

- $\psi \mathrm{e}=$ target elevation angle in receiver coordinates,

- $\lambda=$ wavelength,

- $\quad L=$ propagation loss,

- De = transmitter to target distance,

- $D r=$ target to receiver distance,

- $\sigma=R C S$ of the target.

The transmitting and receiving antennas are omnidirectional in azimuth (horizontal plane from $0^{\circ}$ to $360^{\circ}$ ).

It can be written that:

$G e(\theta e, \psi e)=G e(\psi e)$ and $G r(\theta r, \psi r)=G r(\psi r)$.

The gain of the antennas depends only of the elevation angle of the target (in the vertical plane from $0^{\circ}$ to $+90^{\circ}$ ).

Considering all the realized detections of airliners flying in the area during the campaign of measurements, the average values observed for the elevation angles were:

$$
\begin{aligned}
& \psi e=25^{\circ} \\
& \psi r=30^{\circ}
\end{aligned}
$$

In this slice of elevation angles, the gain of antennas are taken as $G e(\psi e)=G r(\psi r)=1$. 
The RCS of the target $(\sigma)$ can now be calculated from the radar equation knowing the different parameters:

$$
\begin{aligned}
& P e=200 \mathrm{~W} \\
& \lambda=2.6 \mathrm{~m} \\
& L=1 \text { (no loss) } \\
& D e(t)=d 1(t) \text { (from ADS-B trajectory) } \\
& D r(t)=d 2(t) \text { (from ADS-B trajectory) }
\end{aligned}
$$

The evolution of the RCS is then plotted in $\mathrm{dBm}^{2}$ as a function of time (Figure 12), calculated from the previous results of the extraction of the detected plots.

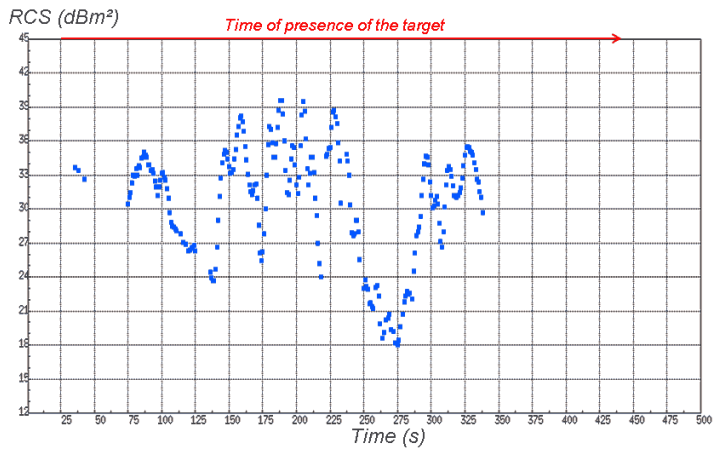

Figure 12 - Evolution of the RCS calculated from radar equation during the passage of the target A321 SWR198F

4) Presentation of the results according to the attitude In the case of a bistatic radar, the target is seen under its attitude angles in azimuth $\theta$ and in elevation $\psi$ which are expressed $\left(\theta_{T x}, \psi_{T x}\right)$ in the transmitter reference and $\left(\theta_{R x}, \psi_{R x}\right)$ for the receiving.

The bearing angles $\theta_{T x}$ and $\theta r$ correspond to the attitude of the target in the horizontal plane:

- $0^{\circ}$ corresponds to front view,

- $90^{\circ}$ is the cross-section view,

- $180^{\circ}$ is rear view with the assumption that the target is symmetrical in the horizontal plane.

The elevation angle $\psi e$ and $\psi r$ corresponds to the attitude of the target in the vertical plane:

- $-90^{\circ}$ is the view from above,

- $0^{\circ}$ is the view on the edge,

- $\quad+90^{\circ}$ is the view from under.

In the case of a monostatic radar, the RCS of the target is a function of the two parameters $\theta$ and $\psi$ because in this case all azimuths are same $\theta=\theta_{T x}=\theta_{R x}$ and all elevation angles too: $\psi$ $=\psi_{T x}=\psi_{R x}$. Thus the results are generally presented on a 2dimensional image $(\theta, \psi)$ with the color as the level of RCS.

For a bistatic radar, the RCS of a target is now a function of four parameters $\theta_{T x}, \psi_{T x}, \theta_{R x}, \psi_{R x}$. It is then difficult to present results in a synthetic way in a 4-dimensional space. In order to simplify, we choose to present them in the two separated references respectively at transmitter site and at receiver site as follows:
- $\theta_{T x}$ and $\psi_{T x}$, the attitude angles under which the target is illuminated,

- $\quad \theta_{R x}$ and $\psi_{R x}$, the angles under which the signal of the target is received.

The following figures show the evolution curves of the azimuth (the blue scale on the left) and of the elevation (the right scale in red) in the transmitter and the receiver sites as references.

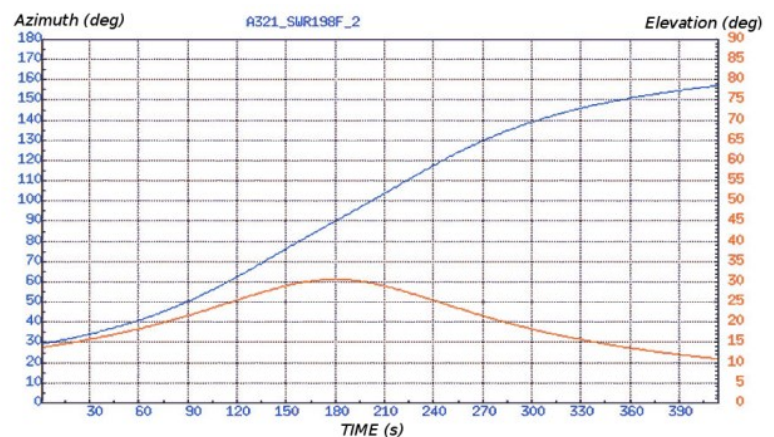

Figure 13 - Variation of attitude angles the target in the transmitter landmark Left: azimuth $\theta_{T x}$ (blue) / right: elevation $\psi_{T x}$ (red)

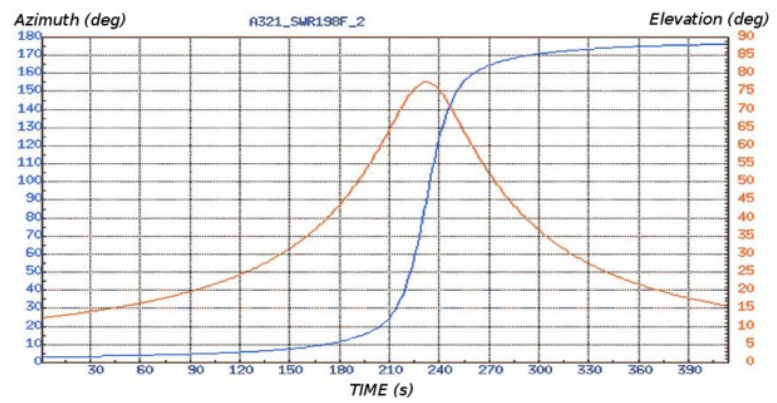

Figure 14 - Variation of attitude angles of the target at receiving Left: azimuth $\theta_{R x}$ (blue) / right: elevation $\psi_{R x}$ (red)

The following figure shows the curves of difference between transmitting and receiving for each angular parameter.

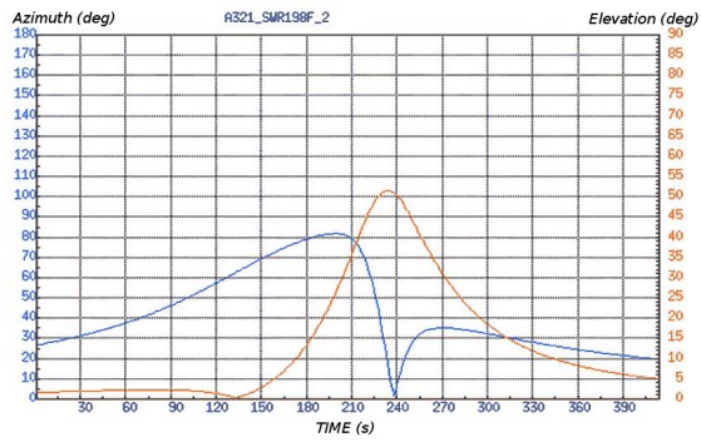

Figure 15 - Difference between attitude angles of the target in transmitting and receiving sites references - Left: azimuth (blue) / right: elevation (red)

It can be noticed that at each time we observe a large difference between attitude angles along the two components. The difference is lower when the plane is at great distances (at the beginning and at the end of the pattern) and the gap becomes more important when the distance decreases between the target and the transmitter or receiver. 
As we got the information of the attitude of the target along the two parameters azimuth and elevation in both landmarks of transmitter and receiver sites, we can then draw the evolutions of the RCS of the target in these two angular references (Figure $16 \&$ Figure 17).

It is recalled that the azimuth angle corresponds to the attitude of the target in the horizontal plane:

- $0^{\circ}$ for front view,

- $90^{\circ}$ is the cross-section view,

- $180^{\circ}$ is the rear view with the assumption that the target is symmetrical in the horizontal plane.

And the angle of elevation corresponds to the attitude of the target in the vertical plane:

- $\quad-90^{\circ}$ is the view from above,

- $0^{\circ}$ is viewed on the edge,

- $\quad+90^{\circ}$ is the view from under.

We present the results obtained for this Airbus A321 for the flight configuration SWR198F presented before.

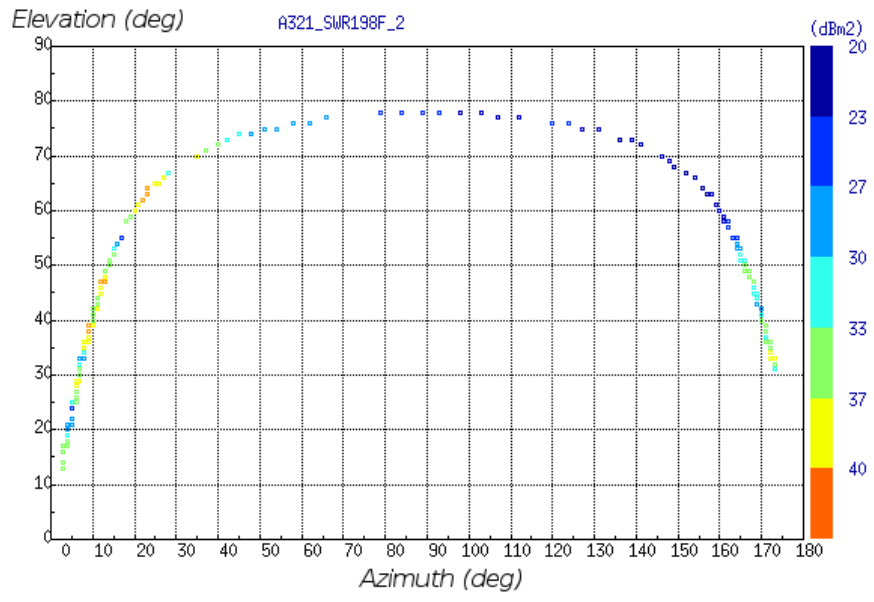

Figure 16 - RCS level (color bar) in function of attitude angles of the Airbus A321 SWR198F viewed from the receiver site

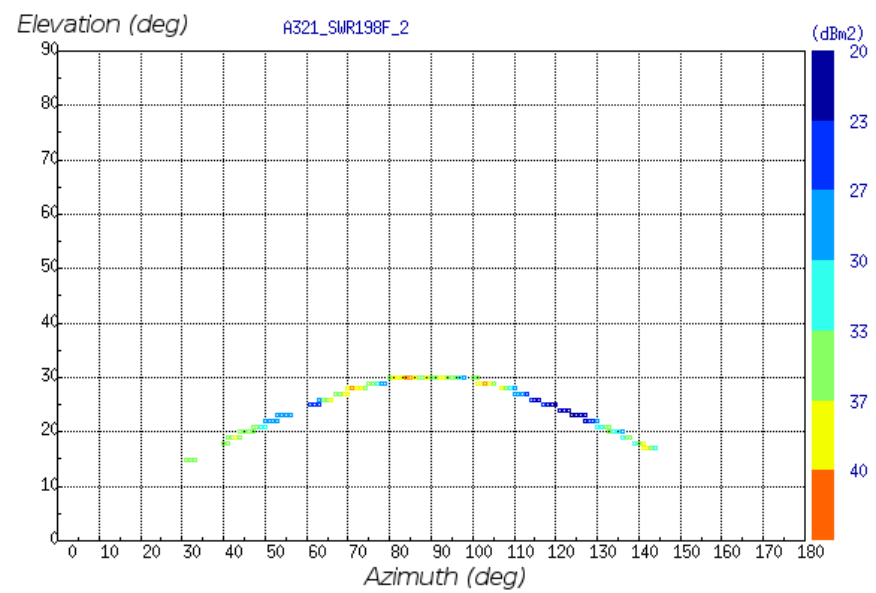

Figure 17 - RCS level (color bar) in function of attitude angles of the Airbus A321 SWR198F viewed from the transmitter site
Due to the bistatic configuration, it can be noticed that there is a large disparity of the levels of RCS measured according to the choice of the reference which is either the transmitter site or the receiver site.

For this configuration with an Airbus A321, the extreme values of RCS which have been measured are:

- $18 \mathrm{dBm}^{2}$ minimum

- $40 \mathrm{dBm}^{2}$ maximum.

Finally, the average value of the measured RCS is around $30 \mathrm{dBm}^{2}$ in the range of elevation angle between $15^{\circ}$ to $75^{\circ}$.

During this campaign of measurements, many other airliners were used as targets of opportunity. The list of airplanes which have been analyzed during their flights with the same conditions is constituted of:

- 3 Airbus A321,

- 3 Airbus A320,

- 5 Airbus A319,

- 3 Boeing 737.

These airplanes of slightly different sizes belong to the same class of medium-haul aircraft. Globally, for all these planes, the average value of the measured RCS is same order of magnitude, around $30 \mathrm{dBm}^{2}$. All these measurements were obtained for elevation angles between $15^{\circ}$ and $75^{\circ}$ and the planes were always seen from below.

These values were compared to the measurements obtained in an anechoic chamber with a downsize model of an equivalent commercial aircraft. The configuration of this measure was azimuth scan at the same level of the model which corresponds to an aspect angle of elevation of $0^{\circ}$. The average value of the RCS was around $25 \mathrm{dBm}^{2}$.

Even though we must be careful with the interpretation of bistatic measurements of the radar cross section, the results deduced from the detection with VOR signal seem to be roughly satisfying with this technique based on the principle of passive radar.

\section{CONCLUSION}

It has been shown that a passive radar using the signal of a source of opportunity could be used as a bistatic base to evaluate the radar signature of a target in large scale of angular domain when it flights in the field of detection of the system.

For a further experiment, a decrease of the distance between the reception base and the transmitter would approach the case of a monostatic radar for which the configuration would be easier to manage for the analysis of the results, especially for the calculation of the RCS in function of the aspect angle.

\section{REFERENCES}

[1.] Azarian S., Lesturgie M., " Revisiting VOR transmitters : MIMO passive radar using legacy infrastructure", 2014 International Radar Conference, Lille, France, September 24-28 (2014). 